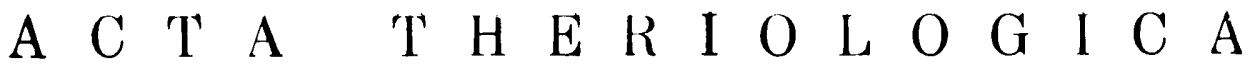

VOL. XII, 16: 253-279.

BIAEOWIEŻA

30.VII.1967

\author{
Stanisław S Z T E Y N
}

\section{Topoyraphie und Kernbau des Mittelhirnes des Sumpibibers}

\author{
[Mit Tafeln X-XIII]
}

\begin{abstract}
Die Untersuchungen wurden auf Grundlage der Querschnitte aus dem Gehirn des Sumpfbibers nach Methode Nissl durchgeführt. In vorliegender Arbeit wurden Lage, Gestaltung und Zellbau der Kerne des Mittelhirnes beim Sumpfbiber beschrieben. Nur einige von diesen Kernen weisen grössere Unterschiede im Vergleich mit anderen Säugern auf. Pars dorsalis nuclei caudalis ventralis lemnisci lateralis ist nicht auf Pars dorso-caudalis und Pars dorso-rostralis geteilt. Der Nucleus interpeduncularis ist einheitlich. Im Nucleus linearis lassen sich nur zwei Teile absondern: der anteriore und posteriore. Der vordere Pol von Nucleus nervi trochlearis ist mit den posterioren Pol von Nucleus nervi oculomotorii vereinigt. Nucleus annularis fasciculi iongitudinalis medialis begrenzt den Fasciculus longitudinalis medialis nur von der ventro-medialen Seite. Die schwarze Substanz ist am besten in Pars compacta, aber am schwächsten in Pars lateralis ausgebildet. Der rote Kern weist keine Einteilung in Pars parvo- und magnocellularis auf.
\end{abstract}

\section{EINLEITUNG}

Vorliegende Arbeit, deren Zweck in der Erkenntnis der Gestaltung, der Lage und des Zellbaues der Kerne des Mittelhirnes des Sumpfbibers war, ist eine Fortsetzung der Untersuchungen des Autors über das Gehirn dieses Tieres. In den vorhergehenden Publikationen (S z t e y n, 1963, 1964, 1965, 1966) wurden die Topographie und der Kernbau der Medulla oblongata und der Kleinhirns des Sumpfbibers beschrieben.

Der innere Bau des Mittelhirnes war der Gegenstand vieler Untersuchungen, die eine ziemlich genaue Erkenntnis der Struktur dieses Gehirnteiles beim Menschen und einigen Säugetierarten ermöglichten. Gleichzeitig haben die Untersuchungen bewiesen, dass das Mesencephalon, trotz verhältnismässig ungrosser Veränderungen, die sich in diesem Gehirnteil in der onto- und filogenetischen Entwicklung und dem ziemlich einfachen Bau vollziehen, gleichzeitig in seiner Struktur bedeutende Unterschiede aufweist. Diese Unterschiede treten nicht nur zwischen dem Mittelhirn der Säuge- und anderer Wirbeltiere auf, aber auch zwischen den einzelnen Arten der Säugetiere. Aus diesem Grunde scheint mi: die Durchführung von 
Untersuchungen zweckmässig zu sein, die als Ziel die Erkennung der Lage und des Baues der Nervenzentren des Mittelhirnes bei denjenigen Tierarten haben, bei denen sie bisher nicht beschrieben worden sind. Ergebnisse derartiger Untersuchungen vergrössern das anatomische Vergleichsmaterial, aber es ist auch möglich, dass sie neue Angaben liefern könnten, die das Ziehen von weiteren Folgerungen gestatten würden betreffs Tätigkeit der einzelnen Nervenzentren des Mittelhirnes. Vorliegende Erwägungen waren für mich der Grund zur Aufnahme von Untersuchungen über die Kerne des Mittelhirnes des Sumpfbibers, von denen es in der Weltliteratur gänzlich an Angaben fehlt.

\section{MATERIAL UND METHODE}

Die Untersuchungen wurden auf 3 Gehirnen von 11 Monate alten Sumpfbibern durchgeführt. Das Material wurde in Äthylalkohol fixiert, dann in Paraffin gebettet und in Querschnitte von $15 \mu$ Stärke geschnitten. $\mathrm{Zu}$ Untersuchungen wurden alle Querschnitte benutzt. Sie wurden mit Methylenblau von Löffler nach der Methode von Nissl gefärbt. Die Kernbeschreibung wurde von posterior in anteriorer Richtung durchgeführt.

\section{ERGEBNISSE}

1. Pars ventro-medialis nuclei caudalis ventralis lemnisci laterali.: (pvm - Abb. 1 u. 9) ist unter dem Zusammenspiel der Kerne des lateralen Streifens am weitesten posterior vorgeschoben. Der beschriebene Kern ist ein deutlicher Zellstreifen, der sich auf der Strecke von der in ungrosser Entfernung posterior vom posterioren Pol des Nucleus latero-dorsalis tegmenti durchgeführten Querfläche, bis zur mit dem posterioren Pol des Nucleus dorsalis raphe tangierenden Querfläche entlang zieht.

Auf den Querschnitten ist der hintere Pol des Kernes eine kleine ausgedehnte Zellgruppe, die medial vom Nucleus subtrigeminalis über dem lateralen Rand der Brückenkerne gelegen ist. In anteriorer Richtung vergrössert sich der Querschnitt des Kernes schnell und nimmt eine abgerundete Gestaltung an. In der halben Länge, vorn vom anterioren Pol des beweglichen Kernes des $N$. trigeminus und des Nucleus subtrigemiralis nimmt der beschriebene Kern eine verlängerte Form einer fast senkrecht gelegenen Zellgruppe an. Gleichzeitig verschiebt sich der Kern stufenweise in dorso-lateraler Richtung und ordnet sich in der Nähe von Lemniscus lateralis auf seiner medialen Seite an. Von der posterioren Grenze im $1 / 4$ ihrer anterioren Länge verringert sich Pars ventro medialis nuclei caudalis ventralis langsam und unterliegt allmählich dem Schwund. Der vordere Pol des Kernes ist auf dem Querschnitt als kleine ausgedehnte Zellgruppe sichtbar, die auf der medialen Seite von Lemniscus lateralis, dorsal und lateral von den Kernen der Brücke, aber ventral und lateral vom Nucleus mesencephalicus prof gelegen ist.

Den Kern bilden sich intensiv färbende, gedrängt angeordnete, spin- 
delförmige und abgerundete Zellen, aber auch wenige dreieckige und vielpolige mit Ausmassen von 12 bis 35 ". Der Zellkern ist kugelig, undeutlich mit zentral situiertem Kernkörperchen. In den Zellen tritt eine grosse Menge tigroidaler Substanz auf und dies in Gestalt von gleichmässig verstreuten feinen Körrichen.

2. Pars dorsalis nuclei caudalis ventralis lemnisci lateralis ( $\mathrm{pd}-\mathrm{Abb}$. 1 u. 10) ist ein weniger deutlicher und fast um die Hälfte kürzerer Zellstreifen als der ventro-mediale Teil dieses Kernes. Der kaudale Pol von Pars dorsalis liegt in geringer Entfernung posterior von der durch den pcsterioren Pol des Nucleus dorsalis raphe durchgeführten Querfläche. Vorn unterliegt der Kern auf der Höhe des posterioren Poles von Nucleus medialis prof. dem Schwund.

Auf den Querschnitten ist der posteriore Abschnitt des Kernes eine ziemlich deutliche ovale Zellgruppe, die auf der lateralen Seite von Lemniscus laterulis gelegen ist, aber dorsal und lateral liegt sie vom vorderen Abschnitt der Pars ventro medialis nuclei caudalis ventralis lemnisci iateralis. Vorn vom vorderen Pol der Pars ventro-medialis ordnen sich die Kernzellen auf einer grösseren Fläche an und der Kern wird weniger zusammengedrängt und undeutlich. Der mittlere und vordere Abschnitt der Pars dorsalis ist von der dorsalen Seite mit Nucleus dorsalis lemnisci lateralis benachbart. In der Nähe des vorderen Poles zerstreuen sich die Kernzellen stufenweise und der Kern unterliegt allmählich auf der Höhe des posterioren Poles von Nucleus medialis profundus dem Schwund. Der vordere Pol von Pars dorsalis wird durch zerstreute, vereinzelte Zellen gebildet, die ventral vom dorsalen Kern der lateralen Schleife liegen.

Der Kern wird durch runde und ovale aber auch unzahlreiche lose angeordnete vielpolige und spindelförmige Zellen mit Ausmassen vor 15 bis $35 \mu$ gebildet. Der Zellkern ist kugelig, undeutlich und hat ein zentral situiertes Kernkörperchen. Die Zellen enthalten unzahlreiche, ziemlich dicke Körnchen von tigroidaler Substanz. Zwischen den Zellen von Pars dorsalis treten verhältnismässig sehr zahlreiche Gliazellen auf.

3. Der Nucleus dorsalis lemnisci lateralis (ndl - Abb. 2 u. 11) ein ziemlich kurzer Streifen von lose angeordnoten Zellen ist in dem dorso-lateralen Teil des Deckels gelegen und zwar lateral von Lemniscus lateralis aber dorsal von der Pars dorsalis nuclei caudalis ventralis lemnisci iateralis.

Auf den Querschnitten ist der posteriore Pol des beschriebenen Kernes eine kleine unregulär geformte Gruppe von zerstreuten Zellen, die lateral von dem dorsalen Teil der lateralen Schleife und des marginalen Kernes des vorderen Pedunculus des Cerebellum und dorsal von Pars dorsalis nuclei caudalis ventralis lemnisci lateralis gelegen ist. Vorn 
wächst die Zellanzahl im Kern an und er vergrössert sich in ventraler Richtung. Die Kernzellen ordnen sich sehr lose und unregulär an, was zur Folge hat, dass seine Gestalt schwer zu beschreiben ist. Im Mittelabschnitt des Kernes ordnen sich die Zellen im Bereich vom inferioren Kand der grauen Substanz des posterioren Colliculus bis zum dorsalen Rand von Pars dorsalis nuclei caudalis ventralis an. In vorderer Richtung verringert sich der Kern allmählich und unterliegt von ventraler Seite dem Schwund. Den vorderen Pol des dorsalen Kernes der lateralen Schleife bilden einzelne zerstreute Zellen, die lateral von Lemniscus lateralis unter der grauen Substanz des posterioren Colliculus liegen.

Den Nucleus dorsalis lemnisci lateralis bilden ziemlich intensiv gefärbte dreieckige und vielpolige aber auch unzahlreiche spindelförmige Zellen mit Ausmassen von 25 bis $40 \mu$. Die Zellen enthalten einen kugeligen, gut sichtbaren Kern mit einem zentral situierten Kernkörperchen. Die tigroidale Substanz tritt in Gestalt von feinen und mittelgrossen Körnern auf und ist gleichmässig im Zellplasma verteilt. Die Zellen des beschriebenen Kernes färben sich etwas dunkler als diejenigen von Pars dorsalis nuclei caudalis ventralis lemnisci lateralis.

4. Der Nucleus ventralis rostralis lemnisci lateralis (nvr - Abb. 3, 4 u. 12) ist der am weitesten nach vorn vergeschobene von dem Zusammenspiel der Kerne von Lemniscus lateralis. Er ist ein deutlicher ziemlich langer Zellstreifen, der fast unter der Oberfläche der lateralen Wand des Mittelhirnes, lateral von Lemniscus lateralis gelegen ist. Nucleus ventralis rostralis zieht sich im Bereich von der Querfläche entlang, die in geringer Entfernung posterior vom vorderen Pol des Nucleus medialis prof. bis zur, mit dem posterioren Pol von Pars reticularis substantiae nigrae tangierenden Querfläche geführt wird.

Auf den Querschnitten ist der posteriore Pol des beschriebenen Kernes eine kleine vertical-ovale Zellgruppe, die an der lateralen Oberfläche des Mittelhirnes, lateral von Lemniscus lateralis aber dorsal und lateral vom Pedunculus cerebri gelegen ist. In vorderer Richtung vergrössert sich der Kern und nimmt die Gestalt einer verlängerten Zellgruppe an, die parallel zur lateralen Oberfläche des Mittelhirnes gelegen ist. Von der halben Länge an verschiebt sich der Kern stufenweise in ventraler Richtung. In der Nähe des vorderen Poles von Nucleus ventralis rostralis nimmt er auf den Querschnitten die Gestalt einer abgerundeten Zellgruppe an. Den vorderen Kernpol bilden vereinzelte Zellen, die über dem dorsalen Rand des Pedunculus cerebri in seiner Nähe gelegen sind.

Den Nucleus ventralis rostralis bilden sich schwach färbende, dicht angeordnete dreieckige und vielpolige aber auch unzahlreiche spindelförmige Zellen mit Ausmassen von 15 bis $25 \mu$, mit einem gut sichtbaren Kern mit einem zentral situierten Kernkörperchen. Die tigroidale Sub- 
stanz tritt in den Zellen in Gestalt von unzahlreichen groben Körnern auf. Zwischen den Zellen des beschriebenen Kernes tritt eine verhältnismässig grosse Anzahl von Gliazellen auf.

5. Nucleus dorsalis tegmenti (ndt - Abb. 1, 2 u. 13) ist ein langer stellenweise nicht besonders deutlicher Zellstreifen, der in dem ventro-medialen Teil der Substantia grisea centralis gelegen ist. Nucleus dorsalis tegmenti zieht sich im Bereich von der Umgebung des posterioren Poles des motorischen Kernes von Nervi trigemiri bis zur in ungrosser Entfernung nach vorn vom posterioren Pol von Nucleus medialis prof. durchgeführten Querfläche entlang. Der posteriore Teil des beschriebenen Kernes liegt dorsal und medial von Nucleus motorius $n$. trigemini und medial von Nucleus mesencephalicus radicis $n$. trigemini. Die vordere Hälfte des Nucleus dorsalis tegmenti ordnet sich an der medialen Seite von Nucleus latero-dorsalis tegmenti an. Der letztens erwähnte Kern teilt den Nucleus dorsalis tegmenti von Nucleus mesencephalicus radicis $n$. trigemini ab.

Auf den Querschnitten ist der posteriore Teil des Kernes eine kleine horizontal-ovale Zellgruppe, die bei Kammer IV, in der Nähe der medialen Fläche gelegen ist. In vorderer Richtung vergrössert sich der beschriebene Kern und nimmt den ganzen ventro-medialen Teil der grauen periventrikulären Substanz ein. Auf der Höhe des posterioren Poles von Nucieus dorsalis raphe wird der Nucleus dorsalis tegmenti mehr kompakt und deutlich. Auf diesem Abschnitt hat der Kern auf den Querschnitten die Gestalt einer abgerundeten Gruppe von dicht angeordneten Zellen, die zwischen dem Nucleus dorsalis raphe und Nucleus dorsalis tegmenti situiert ist. Im vorderen Abschnitt verschiebt sich der Kern in ventraler Richtung unwesentlich und ordnet sich über dem Fasciculus longitudinalis med. an. In der Nähe des vorderen Poles verringert sich der Kern schnell und unterliegt scharf in ungrosser Entfernung vorn vom posterioren Pol des Nucleus medialis profundus dem Schwund.

Den Nucleus dorsalis tegmenti bilden dreieckige und abgerundete Zellen mit Ausmassen von 10 bis $20 \mu$. Die Zellen enthalten unzahlreiche, feine Körner von tigroidaler Substanz. Der Zellkern ist kugelig, undeutlich mit einem zentral situierten Kernkörperchen. Die Zellen von Nucleus dorsalis tegmenti färben sich weniger intensiv als diejenigen von Nucleus latero-dorsalis tegmenti.

6. Nucleus mesencephalicus radicis $n$. trigemini ( $\mathrm{nm}-\mathrm{Abb} .1-8 \mathrm{u}$. 14) ist der längste Kern des Mittelhirnes. Posterior beginnt er im Bereich der Brücke, in der halben Länge des motorischen Kernes von $N$. trigemini. Anterior reicht der beschriebene Kern bis zum Mittelhirn. Sein vorderer Pol liegt minimal in posteriorer Richtung vom vorderen Pol des Nucleus ruber. Der Kern ist deutlich, da sich seine Zellen von der 
Umgebung durch eine charakteristische Gestalt aber auch durch eine viel intensivere Färbung unterscheiden.

Der posteriore Abschnitt von Nucleus mesencephalicus radicis n. trigemini liegt dorsal vom motorischen Kern d. N. trigemini, medial von Nucleus marginalis pedunculi cerebelli oralis und lateral von Nucleus latero-dorsalis tegmenti. In diesem Abschnitt besteht der Kern aus der grössten Zellenanzahl, die auf den Querschnitten sich in eine ausgedehnte Gruppe von unregulärer Gestalt anordnen. In vorderer Richtung verringert sich die Anzahl der Zellen im beschriebenen Kern und sie ordnen sich längs der äusseren Fläche der grauen, periventrikulären Substanz an. Im Bereich des Mittelhirnes ordnen sich die Zellen des Nucleus mesencephalicus radicis $n$. trigemiri in eine längliche Reihe an, längs des lateralen Randes der grauen, periventrikulären Substanz. Solch ein Kernbild erhält sich bis zu seinem anterioren Pol. Auf den Querschnitten ist der Kern in diesem Abschritt durch einige bis 12-17 Zellen repräsentiert.

Den erwähnten Kern bilden sich intensiv färbende ovale, abgerundete Zellen mit Ausmassen von 30 bis $55 \mu$. Der Zellkern ist kugelig, undeutlich mit einem zentral situierten Kernkörperchen. Die Zellen sind mit einer grossen Menge von tigroidaler Substanz in Gestalt von mittelgrossen Körnern ausgefüllt.

7. Nucleus marginalis pedunculi cerebell oralis (npc - Abb. 1 u. 15) ist ein langer gut ausgebildeter Zellstreifen, der den Pedunculus cerebelli oralis umwindet. Dieser Kern zieht sich von der in ungrosser Entfernung nach vorn vom posterioren Pol des Nucleus mesencephalicus ra(iicis n. trigemini durchgeführten Querfläche, bis zur mit dem posterioren Pol von Nucleus medialis profundus tangierenden Querfläche.

Auf den Querschnitten ist die Gestalt des Kernes veränderlich. In der Nähe des posterioren Poles hat er die Gestalt einer verlängerten Zellgruppe, die lateral von Nucleus mesencephalicus radicis $n$. trigemini und dorsal von Nucleus motorius $n$. trigemini gelegen ist. Auf diesem Abschnitt begrenzt der Kern den Pedunculus cerebelli oralis von der medialen und ventro-medialen Seite. In vorderer Richtung vergrössert sich der Kern und nimmt auf den Querschnitten die Gestalt eines schmalen schräg anliegenden Hufeisens an, das den vorderen Pedunculus des Cerebellums umfasst. Das Joch des Hufeisens ist ventral und lateral gerichtet und die dorso-medial gerichteten offenen Scherkel stützen sich auf dèn Boden von Kammer IV. Die Zellen im Kern sind ungleichmässig angeordnet, kompakter in den Schenkeln, loser im Joch des Hufeisens. Vor der halben Länge des beschriebenen Kernes verringert sich sein inferiorer Schenkel stufenweise und unterliegt von der Seite der Kammer dem Schwund, dagegen vorgrössert sich der auf der dorso-lateralen Sei- 
te von Pedunculus cerebelli oralis gelegene Teil des Kernes. Auf der Höhe der posterioren Grenze, in einem Drittel des vorderen Teiles des Kernes hat er die Gestalt einer verlängerten, bogenförmigen Zellgruppe, die den anterioren Pedunculus des Cerebellum von der dorso-lateralen Seite umwindet. In anteriorer Richtung verringert sich die Anzahl der Zellen im Kern aber auch sein Querschnitt vermindert sich und er verschwindet langsam auf der Höhe des posterioren Poles von Nucleus medialis prof., auf der lateralen Seite von Nucleus mesencephalicus radicis n. trigemini.

Den Nucleus marginalis pedunculi cerebelli oralis bilden spindelförmige und verhältnismässig unzahlreiche vielpolige und dreieckige Zellen mit Ausmassen von 10 bis $30 \mu$. Die Zellen enthalten unzahlreiche feine Körner von tigroidaler Substanz und einen grossen, kugeligen gut sichtbaren Kern mit einem zentral situierten Kernkörperchen. Die Zellen des beschriebenen Kernes färben sich bedeutend weniger intensiv als diejenigen von Nucleus mesencephalicus $n$ trigemini.

8. Nucleus latero-dorsalis tegmenti (ldt - Abb. 1, 2 u. 16) ist ein langer nicht besonders deutlicher Zellstreifen, der im vorderen Teil der Brücke und im posterioren Teil des Mittelhirnes, lateral von Nucleus dorsalis tegmenti aber medial und ventral von Nucleus mesencephalicus radicis $n$. trigemini gelegen ist. Posterior beginnt der beschriebene Kern in der halben Länge von Nucleus dorsalis tegmenti. Der anteriore Pol von Nucleus latero-dorsalis tegmenti liegt auf der Höhe der Querfläche; die in der halben Länge von Nucleus medialis prof. geführt wird.

Auf den Querschnitten ist der posteriore Pol des Kernes eine kleine unregulär gestaltete Zellgruppe, die an Kammer IV gelegen ist, dorsal und medial vom motorischen Kern d. $N$. trigemini, in der Nähe der medialen Fläche von Nucleus mesencephalicus radicis $n$. trigemini. Vorn wächst die Anzahl der Zellen im Kern an, und der Kern vergrössert sich in medialer Richtung und füllt die ganze Fläche zwischen Nucleus dorsalis tegmenti und Nucleus mesencephalicus radicis $n$. trigemini aus. Der Kern breitet sich ebenfalls in ventraler Richtung aus und sein inferiorer Teil ordnet sich unterhalb der grauen preventrikulären Substanz an. Der anteriore Abschnitt des beschriebenen Kernes ist undeutlicher. Seine Zellen zerstreuen sich auf einer ziemlich grossen ventro-medialen Fläche der grauen preventrikulären Substanz, medial von Nucleus mesencephalicus radicis $n$. trigemini und lateral von Nucleus dorsalis raphe. Der Kern unterliegt allmählich, stufenweise in der Umgebung der halben Länge von Nucleus medialis prof. dem Schwund.

Den Nucleus latero-dorsalis tegmenti bilden lose und unregulär angeordnete verschiedengestaltige Zellen mit Ausmassen von 15 bis $40 \mu$, mit zahlreichen, feinen Körnen der tigroidalen Substanz und mit einem 
nicht besonders deutlichen Kern, der ein zentral situiertes Kernkörperchen besitzt.

9. Nucleus mesencephalicus profundus ( $\mathrm{mp}$ - Abb. 1-7 u. 17), auch Substantia reticularis mesencephali benannt, ist kein deutlich sich hervortuender Zellstreifen. Er wird grundsätzlich durch die sich nicht absondernde graue Substanz gebildet, die sich in den freien Räumen zwischen anderen Kernen des Mittelhirnes anordnet und nur in einigen Abschnitten des Mittelhirnes nimmt der Nucleus mesencephalicus prof. die Gestalt einer deutlichen Zellenanhäufung an.

Im posterioren Teil des Mittelhirnes wird der Nucleus mesencephalicus prof. durch zerstreute, sich intesiv färbende vielpolige und spindelförmige Zellen mit Ausmassen von 25 bis 60 gebildet, der medial von der lateralen Schleife, ventral von Nucleus mesencephalicus radicis $n$. trigemini und lateral von Nucleus medialis prof. liegt. Dieser Teil des beschriebenen Kernes geht posterior in die Substantia reticularis der Brücke über.

Posterior vom anterioren Pol des Nucleus medialis prof. wächst die Anzahl der Zeller im Kern an. Sie ordnen sich auf einer grossen Flärche auf der ventralen Seite von Area cuneiformis an, aber lateral von Fasciculus longitudinalis med. und dorsal von Pedunculus cerebri und auch von Pars compacta substantiae nigrae. Diesen Abschnitt von Nucleus mesencephalicus profundus bilden Zellen, die der Grösse und Gestalt nach an diejenigen des posterioren Abschnittes angenähert sind, aber von denen sie sich durch eine weniger intensive Färbung unterscheiden.

Im anterioren Abschnitt des Mittelhirnes bilden den Nucleus mesencephalicus prof. sich hell färbende, lose angeordnete, vielpolige und abgerundete aber auch unzahlreiche spindelförmige Zellen mit Ausmassen von 15 bis $30 \mu$. Die Zellen des vorderen Abschnittes des beschriebenen Kernes liegen ventral von der grauen zentralen Substanz, aber dorsal und medial von der kompakten schwarzen Substanz, den Nucleus ruber von der lateralen und dorsalen Seite umfassend. Vorn geht Nucleus nesencephalicus prof. in die graue Substanz des Zwischenhirnes über.

10. Nucleus dorsalis raphe ( $\mathrm{dr}$ - Abb. $2,3 \mathrm{u} .18)$ ist ein langer, schnaler deutlicher Zellstreifen, der preventrikulär in der Nähe der Naht gelegen ist. Posterior erscheint der Kern auf der Höhe der Querfläche, die man in der halben Länge von Nucleus latero-dorsalis tegmenti durchführt. Der vordere Pol von Nucleus dorsalis raphe reicht bis zur mit dem posterioren Pol von Nucleus trochlearis tangierenden Querfläche.

Auf den Querschnitten im posterioren Abschnitt ist der beschriekene Kern eine schmale verlängerte Zellgruppe, die senkrecht an der Niht, auf der Fläche von der Kammer bis zu Fasc. longitudinalis med. gelegen ist. Im Mittelabschnitt ist der Kern kürzer aber breiter - auf den Quer- 
schnitten wird er durch eine unregulär gestaltete Zellgruppe gebildet, die in der halben Entfernung zwischen der Kammer und dem Fasc. longitudinalis med. gelegen ist. Anterior von der halben Länge, nach dem Schwund von Nucleus latero-dorsalis tegmenti, wird der beschriebene Kern weniger kompakt und undeutlicher, und seine Zellen zerstreuen sich auf einer grossen Fläche in der ventro-medialen Partie der grauen periventrikulären Substanz. Im vorderen Abschnitt wird der Kern wiederum deutlicher und kompakter; gleichzeitig verschiebt sich der dorsale Kern der Naht ventral und entfernt sich von der Wasserleitung des Mittelhirnes indem er sich an den Fasc. longitudinalis med. annähert. In der Richtung des vorderen Poles verringert sich der Kern und unterliegt allmählich in der Umgebung des posterioren Poles von Nucleus trochlearis dem Schwund.

Den dorsalen Kern der Naht bilden sich intensiv färbende, ziemlich lose und unregulär angeordnete, dreieckige, spindelförmige und ovale Zellen mit Ausmassen von 15 bis $40 \mu$. Der Zellkern ist gross, kugelig und hat ein zentral situiertes Kernkörperchen. Die tigroidale Substanz tritt in den Zellen in Gestalt von zahlreichen feinen Körnern auf, die sich zeitweise in grössere Klümpfchen anhäufen.

11. Nucleus linearis ist man gewöhnt in drei Teile zu teilen: Pars caudalis, Pars medialis und Pars oralis. Bei dem Sumpfbiber lässt sich eine derartige Einteilung des Nucleus linearis nicht durchführen, denn im $N$. linearis dieses Tieres kann man nur zwei Teile absondern nämlich: den anterioren und kaudalen.

Pars caudalis nuclei linearis (lc - Abb. 2, 3 u. 19) ist ein langer, aber undeutlicher, durchbrochener Zellstreifen, der sich im Bereich von der Querfläche, die in der Umgebung des vorderen Poles von Pars ventro-medialis nuclei caudalis ventralis lemnisci lateralis durchgeführt wird, langzieht, bis zur Querfläche, die sich in kleiner Entfernung posterior vom posterioren Pol des Nucleus trochlearis befindet. Der erwähnte Kern ordnet sich ventral von Fasc. longitudinals med. und dorsal von der grauen Substanz der Brücke (hinteres Segment) und vom Nucleus interpeduncularis (vorderes Segment des Kernes) an. Auf den Querschnitten ist die Gestalt des Kernes unregulär und veränderlich. Nur an einigen Stellen ordnen sich die Zellen des Nucleus linearis pars caudalis so an, wie dies für diesen Kern charakteristisch ist, also linear in der media-len Fläche und an diesen Stellen ist der Kern deutlicher. Häufiger jedoch wird der Kern von zerstreuten Zellen gebildet, die sich im Bereich von unregulärer Gestalt sowohl in der medialen Fläche wie auch in ihrer Nähe anordnen. Im Verlauf des Kernes treten kurze, unreguläre Unterbrechungen auf. In Pars caudalis nuclei linearis bilden sich ziemlich intensiv färbende abgerundete, vielpolige und spindelförmige Zellen mit Aus- 
massen von 10 bis $30 \mu$, mit zahlreichen feinen Körnern der tigroidalen Substanz und einem kugeligen, undeutlichen Kern, der ein zentral situiertes Kernkörperchen enthält.

Pars oralis nuclei linearis (lo - Abh. 5 u. 2C) ist um die Hälfte kleiner als der posteriore Teil und ist ähnlich wie der letzte sehr undeutlich. Pars oralis nuclei linearis ist kein kompakter Kern. Sie hat die Gestalt eines Streifens mit lose angeordneten Zellen, die in der medialen Fläche und in ihrer Nähe liegen, ventral von Fasc. longitudinalis med. und dorsal von Nucleus interpeduncularis und Nucleus interstitialis comissurae Foreli. Der posterior beschriebene Kern erscheint in der halben Länge von Nucleus trochlearis. Der Kern schwindet in kleiner Entfernung nach vorn hin vom posterioren Pol des Nucleus ruber. Auf den Querschnitten ist Pars oralis nuclei linearis ein kleiner unregulär gestalteter Zellstreifen der in der medialen Fläche, ventral von Fasc. longitudinalis med. liegt. Der vordere Kernabschnitt ordnet sich zwischen den roten Kernen der linken und rechten Seite an. Den vorderen Teil von Nucleus linearis bilden sich ziemlich intensiv färbende spindelförmige, vielpolige und abgerundete Zellen mit Ausmassen von 8 bis $30 \mu$. Die Zellen enthalten eine grosse Menge der tigroidalen Substanz in Gestalt von Körnern verschiedener Grösse. Der Zellkern ist kugelig, nicht besonders deutlich und hat ein zentral situiertes Kerinkörperchen. Die Zellen von Pars oralis nuclei linearis färben sich deutlich intensiver als diejenigen von Nucleus interstitialis commissurae Foreli.

12. Nucleus annularis fasciculi longitudinalis medialis (na - Abb. 2 u. 21) ist ein kurzer undeutlicher Zellstreifen, gelegen im posterioren Abschnitt des Mittelhirnes, auf der ventro-medialen Seite von Fasc. longitudinalis med. Kaudal erscheint der erwähnte Kern auf der Höhe des vorderen Poles von Pars ventro-medialis nuclei caudalis ventralis lemnisci lat. Anterior unterliegt der Kern in kleiner Entfernung nach vorn hin von der Querfläche, die durch den anterioren Pol von Nucleus dorsalis tegmenti geführt wird, dem Schwund.

Auf den Querschnitten bilden den erwähnten Kern unzahlreiche an der Naht liegende Zellen, in der Nähe des ventro-medialen Randes von Fasc. longitudinalis med. Der Kern liegt dorsal von Pars caudalis nuclei linearis und ventral von $N$. dorsalis raphe. Die vordere Hälfte des Kernes ordnet sich dorsal und medial von $N$. medialis prof. an. Auf einigen Präparaten tangiert der dorsale Rand von Nucleus annularis fasc. longitudinalis med. mit dem ventralen Rand von Nucleus dorsalis raphe. Beide Kerne kann man jedoch auseinander grenzen, da ihre Zellen sich durch die Gestalt und die Intensität der Färbung unterscheiden.

Den Nucleus annularis des Fasc. longitudinalis med. bilden ziemlich lose angeordnete, sich intensiv färbende, abgerundete und ovale Zellen 
mit Ausmassen von 15 bis $20 \mu$. Der Zellkern ist kugelig, undeutlich und enthält ein zentral situiertes Kernkörperchen. Die Zellen sind mit zahlreichen, feinen Körnern der tigroidalen Substanz ausgefüllt. Die Zellen des beschriebenen Kernes färben sich intensiver als diejenigen von $\mathrm{Nu}$ cleus dorsalis raphe.

13. Nucleus medialis profundus (nmp - Abb. 2, 3 u. 22) ist ein larger deutlich von der Umgebung abgegrenzter Zellstreifen, der im posterioren Teil des Mittelhirnes auf der ventro-lateralen Seite von Fasciculus longitudinalis medialis gelegen ist. Der kaudale Teil des erwähnten Kernes liegt in der halben Länge von Nucleus mesencephalicus radicis $n$. trigemini. Vorn reicht der Nucleus medialis prof. zur Querfläche, die durch den posterioren Pol des Nucleus interpeduncularis geführt ist.

Auf den Querschnitten hat der Kern die Gestalt eines Ovales, das schräg von der dorso-lateralen nach der ventro-medialen Seite in kleiner Entfernung von der medialen Fläche gelegen ist. Nur in der Nähe des anterioren und posterioren Poles ist der Umriss des Kernes abgerundet.

Den Nucleus med. prof. bilden gleichmässig angeordnete, sich ziemlich intensiv färbende dreieckige und abgerundete oder einzelne spindelförmige Zellen mit Ausmassen von 15 bis $30 \mu$. Die Zellen enthalten einen grossen, kugeligen, gut sichtbaren Kern mit einem zentral situierten Kernkörperchen. Tigroidale Körner von verschiedener Grösse sind gleichmässig angeordnet und weisen eire Tendenz zur Anhäufung auf der Peripherie auf.

14. Area cuneiformis (ac - Abb. 3 u. 23) ist ein ziemlich langer etwas undeutlicher Zellstreifen, der an den ventro-lateralen Teil der grauen periventrikulären Substanz anliegt. Der posteriore Pol des beschrieberien Kernes erscheint auf der Höhe der Querfläche, die in kleiner Entfernung nach vorn hin vom posterioren $\mathrm{Pol}$ des Nucleus medialis prof. geführt ist. Der anteriore Pol von Area cuneiformis reicht bis zur, mit dem posterioren Pol von Nucleus trochlearis tangierenden Querfläche.

Auf den Querschnitten ist die Gestalt des Kernes nur im mittleren und vorderen Abschnitt mit seinem Namen übereinstimmend. In diesen Abschnitten hat die Area cuneiformis die Gestalt eines Keiles, dessen Basis zur grauen periventrikulären Substanz gerichtet ist, aber mit dem Gipfel ventral und lateral. Im posterioren Abschnitt hat der erwähnte Kern auf den Querschnitten die Gestalt eines unregulären Viereckes. Sowohl von der grauen preventrikulären Substanz, die auf der medialen Seite des beschriebenen Kernes liegt, wie auch von der grauen Substanz des Colliculus post., der mit seiner dorsalen Seite benachbart ist, ist die Area cuneiformis vermittels einer dünnen aber deutlichen Schicht von der weissen Substanz abgeteilt. Auf ihrer ganzen Länge ist die Area cunei- 
formis von der medialen Seite mit dem längs der Fläche der grauen periventrikulären Substanz liegenden Nucleus mesencephalicus radicis $n$. trigemini benachbart.

Die Area cuneiformis bilden lose, unter den Fasern untergebrachte, sich schwach färbende spindelförmige aber auch unzahlreiche dreieckige und vielpolige Zellen mit Ausmassen von 10 bis $20 \mu$. In den Zellen tre.ten unzahlreiche Körner von mittlerer Grösse der tigroidalen Substanz auf. Der Zellkern ist kugelig, undeutlich mit einem zentral situierten Kernkörperchen. Ausser den oben erwähnten Zellen trifft man im beschriebenen Kern ebenfalls vereinzelte dreieckige und vielpolige Zellen mit Ausmassen, die bis $40 \mu$ ausmachen.

15. Nucleus interpeduncularis ( $\mathrm{ni}-\mathrm{Abb} .4-6 \mathrm{u}$. 24) ist ein langer deutlicher Zellstreifen, der in der medialen Ebene, an der ventralen Fläche der Mittelhirnes liegt. Der posteriore Pol des beschriebenen Kernes liegt auf der Höhe der Querfläche, die durch den vorderen Pol von $\mathrm{Nu}$ cleus medialis prof. geführt ist. Vorn schwindet der Nucleus interpeduncularis in der halben Länge von Nucleus ruber. Auf den Querschnitten hat der Nucleus interpeduncularis die Gestalt eines Keiles, dessen Gipfel dorsal gerichtet ist, aber die stark ausgebreitete Basis gelangt bis zur ventralen Fläche des Mittelhirnes und bildet in ihr eine deutliche Wölbung. Nur auf einer kurzen Strecke im posterioren Abschnitt des Kernes hat sein Umriss auf den Querschnitten die Gestalt einer unregulären Zellgruppe, die in einer gewissen Entfernung von der ventralen Fläche des Gehirnes gelegen ist.

Den Nucleus interpeduncularis bilden ziemlich lose und unregulär angeordnete, sich intensiv färbende, spindelförmige, dreieckige und abgerundete Zellen mit Ausmassen von 15 bis $40 \mu$. Der Zellkern ist kugelig etwas undeutlich und enthält ein zentral situiertes Kernkörperchen. Die Zellen ausfüllende tigroidale Substanz hat eine schäumige Konsistenz. Zwischen den oben beschriebenen Zellen treten im Kern zahlreiche Gliazellen auf, die verursachen, dass der Kern die Gestalt eines kompakten, deutlichen Zellstreifens hat.

16. Nucleus nervi trochlearis (nt - Abb. 4 u. 25) ist ein verhältnismässig kurzer aber deutlicher Zellstreifen, der ventral von der Substantia grisea centralis an der dorso-lateralen Fläche von Fasc. longitudinalis med. liegt. Der posteriore Pol des erwähnten Kernes befindet sich auf der Höhe der Querfläche, die durch den kaudalen Pol des kompakten Teiles der schwarzen Substanz geführt ist. Der vordere Teil des Nucleus n. trochlearis kontaktiert mit dem posterioren Pol des Nucleus n. oculomotorii in geringer Entfernung posterior vom kaudalen Pol des Nucleus ruber.

Auf den Querschnitten hat der Nucleus n. trochlearis die Gestalt ova- 
len Zellgruppe, die auf einigen Präparaten eine ziemlich deutliche Einteilung auf den dorsc-lateralen Teil und den ventro-medialen hat.

Den Nucleus $n$. trochlearis bilden ziemlich lose dislozierte, sich intensiv färbende dreicckige und vielpolige Zellen mit Ausmassen von 30 bis $45 \mu$. Die Zellen enthalten zahlreiche grobe Körner der tigroidalen Substanz, aber auch einen grossen, kugeligen, gut sichtbaren Kern mit einem zentral situierten Kernkörperchen.

17. Pars compacta substantiae nigrae (cn - Abb. 4-8, u. 26) ist die am besten ausgestaltete unter den drei Teilen von Substantia nigra. Sie zieht sich von der Querfläche, die durch den vorderen Pol von Nucleus dorsalis raphe bis zum Zwischenhirn geführt ist - ihr vorderer Pol liegt in geringer Entfernung nach vorn hin vom anterioren Pol des Nucleus interstitialis fasciculi longitudinalis medialis. Pars compacta substantiae nigrae ist ein deutlicher, flacher Zellstreifen, der sich auf der Grenze zwischen dem Deckel und der Basis der Pedunculi cerebri befindet.

Auf den Querschnitten ist der beschriebene Teil der Substantia nigra eine bandartige verlängerte, leicht bogenförmig gewölbte Zellgruppe, die schräg von der dorso-lateralen Seite nach der ventro-medialen Seite hin angeordnet ist. Pars compacta substantiae nigrae begrenzt von der dorso-medialen Seite die Basis der Pedunculi cerebri. In der Nähe des anterioren und posterioren Poles, wo sich der Kern verringert und die Gestalt eines verlängerten schräg angeordneten Ovales annimmt, ist er kompakter und deutlicher als im Mittelteil, der aus lose dislozierten Zellen gebildet wird.

Pars compacta substantiae nigrae bilden sich intensiv färbende dreieckige, vielpolige und spindelförmige Zellen mit Ausmassen von 25 bis $40 \mu$. Die Zellen enthalten einen grossen, kugeligen oder ovalen Kern mit einem zentral situierten Kernkörperchen. Die tigroidale Substanz ist in Gestalt von zahlreichen, feinen Körnern in der Mehrzahl der Zellen auf ihrer Peripherie angehäuft. Im posterioren Teil überwiegen dreieckige und vielpolige Zellen im Kern, aber die spindelförmigen sind verhältnismässig unzahlreich. In vorderer Richtung vergrössert sich die Anzahl der spindelförmigen Zellen stufenweise und sie machen die Mehrheit im vorderen Abschnitt von Pars compacta substantiae nigrae aus.

18. Pars reticularis substantiae nigrae ( $\mathrm{rn}-\mathrm{Abb} .5-8 \mathrm{u}$. 27) ist ein deutlicher gut ausgestalteter Zellstreifen, der in der Basis des Pedunculus cerebri, auf der ventro-lateralen Seite von Pars compacta substantiae nigrae gelegen ist. Pars reticularis ist kürzer als Pars compacta. Posterior beginnt sie auf der Höhe der Querfläche, die durch den vorderen Pol von Nucleus n. trochlearis d. i. auf der Höhe der vorderen Grenze eines Sechstels des kaudalen Teiles der Pars compacta durchgeführt ist. Der 
vordere Pol des beschriebenen Kernes liegt in kleiner Entfernung posterior von dem vorderen Pol der Pars compacta.

Auf den Querschnitten bilden der anteriore und posteriore Pol des Kernes unzahlreiche Zellen, die an den ventro-lateralen Rand von Pars compacta in ihrer halben Länge gelegen sind. Im Mittelabschnitt ist die Fars reticularis substantiae nigrae am besten ausgestaltet - ihre Zellen sind auf einem grossen Bereich in der Basis von Pedunculi cerebri, längs des ganzen ventro-lateralen Randes von Pars compacta disloziert. Dic dorso-laterale Fläche von Pars reticularis, an Pars compacta anliegend, ist abgeflacht, die ventro-laterale Fläche dagegen ist gewölbt und ver-läuft ungefähr parallel zur äusseren Fläche des Pedunculus cerebri.

Die Pars reticularis substantiae nigrae bilden, sich intensiv färbende, sehr lose angeordnete vielpolige, dreieckige und spindelförmige, aber auch unzahlreiche abgerundete Zellen mit Ausmassen von 15 bis $40 \mu$. Der Zellkern ist kugelig oder oval, undeutlich mit einem zentral situierten Kernkörperchen. In den Zellen treten zahlreiche feine Körner der tigroidalen Substanz auf. Die Zellen von Pars reticularis sind viel loser angeordnet als diejenigen von Pars compacta, aber die Färbungsintensität der Zellen ist in beiden oben erwähnten Teilen der schwarzen Substanz angenähert.

19. Pars lateralis substantiae nigrae (ln - Abb. $6-8$ u. 28 ) ist der kürzeste und am schwächsten ausgestaltete Teil der schwarzen Substanz. Der beschriebene Kern ist ein nicht besonders deutlicher Zellstreifen, der auf der dorsalen Seite des Mittelabschnittes von Pars compacta gelegen ist. Posterior erscheint der laterale Teil der schwarzen Substanz auf der Höhe der vorderen Grenze eines Drittels des kaudalen Teiles von Pars compacta substantiae nigrae. Der vordere Pol des Kernes reicht bis zur Querfläche, die mit dem vorderen Pol von Nucleus basalis radicis optici kontaktiert.

Auf den Querschnitten ist der posteriore und mittlere Abschnitt des Kernes eine ziemlich grosse Zellgruppe, die in der Nähe der dorsalen Fläche des kompakten Teiles der schwarzen Substanz dorsal und lateral von Nucleus ruber und ventral von der grauen Substanz des Colliculus anterior laminae quadrigeminae gelegen ist. Im vorderen Abschnitt werden die Grenzen des Kernes noch undeutlicher. Seine Zellen ordnen sich stufenweise immer loser an und der erwähnte Teil der schwarzen Substanz unterliegt allmählich auf der Höhe des vorderen Poles von Nucleus basalis radicis optici dem Schwund.

Der laterale Teil der schwarzen Substanz wird aus verschieden gestal- tigen Zellen mit Ausmassen von 25 bis $40 \mu$ gebildet, wobei der schwach sichtbare Kern kugelig oder oval ist. Er enthält ein zentral situiertes Kernkörperchen. Die tigroidale Substanz tritt in Gestalt von ziemlich 
zahlreichen feinen Körnern auf. Im posterioren Abschnitt des beschricbenen Kernes sind die Zellen dichter und regulärer angeordnet als im anterioren Abschnitt. Die Zellen des lateralen Teiles färben sich etwas heller als diejenigen der Pars compacta und der Pars reticularis substantiae nigrae.

20. Nucleus ventralis tegmenti (vt - Abb. 4-6 u. 29), auch Area ventralis tegmenti benannt, ist ein langer, aber undeutlicher Zellstreifen, der im ventralen Teil des Mittelhirnes zwischen dem Nucleus interpeduncularis und der Pars compacta substantiae nigrae gelegen ist. Posterior erscheint der erwähnte Kern in der Umgebung der halben Länge von Nucleus ventralis rostralis lemnisci lat. Vorn schwindet der Nucleus ventralis tegmenti auf der Höhe der mit dem posterioren Pol von Nucleus medianus post. kontaktierenden Querfläche.

Auf den Querschnitten hat der beschriebene Kern eine an das Dreieck angenäherte Gestalt, dessen Basis gipfelwärts, aber der Gipfel selbst ventral gerichtet sind. Der Nucleus ventralis tegmenti ist von der medialen Seite mit Nucleus interpeduncularis, aber von der lateralen mit Pars compacta substantiae nigrae benachbart. Ventral und lateral vom erwähnten Kern ordnet sich der Pedunculus mamillaris an. Auf einer kurzen Strecke ist der Nucleus ventralis tegmenti im Mittelabschnitt in seinem oberen Teil mit dem gleichnamigen Kern der gegenüber liegenden Seite vereinigt, mit dem er über dem dorsalen Rand von Nucleus interpeduncularis kontaktiert. Im anterioren und posterioren Abschnitt sind die Nuclei ventrales tegmenti der beiden Seiten voneinander deutlich abgeteilt.

Den Nucleus ventralis tegmenti bilden sehr lose und unregulär angeordnete sich ziemlich hell färbende dreieckige und spindelförmige Zellen mit Ausmassen von 15 bis $35 \mu$, mit einem deutlichen kugeligen oder ovalen Kern, der ein zentral situiertes Kernkörperchen besitzt. In den Zellen treten nicht besonders zahlreiche regulär angeordnete feine aber auch mittelgrosse Körner der tigroidalen Substanz auf.

21. Nucleus interstitialis commissurae Foreli (nic - Abb. 5, 6 u. 30) ist eine undeutliche Anhäufung von zwischen den Fasern der Commissurae Foreli, aber über dem anterioren Abschnitt von Nucleus interpeduncularis angeordneten Zellen. Der posterior beschriebene Kern erscheint auf der Höhe der Querfläche, die durch den vorderen Pol von Nucleus trochlearis geführt ist. Anterior unterliegt der Kern in der Umgebung der mit dem posterioren Pol von Nucleus medianus post. kontaktierenden Querfläche dem Schwund.

Auf den Querschnitten bilden den Nucleus interstitialis commissurae Foreli lose zerstreute Zellen, die in der Umgebung der medialen Fläche, ventral von Pars oralis nuclei linearis aber dorsal von Nucleus interpe- 
duncularis gelegen sind. Die vordere Hälfte des Kernes liegt von Nucleus ruber ventral und medial.

Den Kern bilden sich hell färbende spindelförmige und vielpolige Zellen mit Ausmassen von 15 bis $35 \mu$. In den Zellen treten wenige rnittelgrosse Körner der tigroidalen Substanz auf. Der Zellkern ist kugelig, gut sichtbar, mit einem zentral situierten Kernkörperchen.

22. Nucleus nervi oculomotorii (nc - Abb. 5-7 u. 31) ist ein von der Ümgebung deutlich abgegrenzter Zellstreifen, der in der Verlängerung in vorderer Richtung von Nucleus $n$. trochlearis gelegen ist. Der postericre Teil des Kernes kontaktiert mit dem vorderen Pol von Nucleus $n$. trochlearis, aber er ist von ihm durch eine deutliche Verschmälerung abgeteilt. Der anteriore Pol von Nucleus $n$. oculomotorii gelangt bis zur Querfläche, die durch die posteriore Grenze eines Drittels des vorderen Teiles von Nucleus ruber geführt ist. Der Nucleus n. oculomotorii ist zweimal länger als Nucleus trochlearis. Der posteriore Abschnitt von Nucleus $n$. oculomotorii liegt an der dorso-lateralen Fläche von Fasc. longitudinaiis med. Vorn ordnet sich der beschriebene Kern immer näher an die mediale Fläche an und nimmt allmählich eine dorsale Lage an, aber danach im vorderen Abschnitt eine dorso-mediale im Verhältnis zu Fasc. longitudinalis med. Trotz Annäherung an die mediale Fläche sind die Nuclei $n$. oculomotories beider Seiten auf der ganzen Länge deutlich voneinander abgeteilt.

Auf den Querschnitten ist der Nucleus n. oculomotorii eine ovale Zellgruppe. Auf einer kurzen Strecke deutet sich im vorderen Abschnitt des Kernes seine Einteilung auf den dorsalen und ventralen Teil markant an.

Den Nucleus nervi oculomotorii bilden dicht angeordnete, sich intensiv färbende, dreieckige und vielpolige Zellen mit Ausmassen von 25 bis $45 \mu$. In den Zellen befindet sich eine grosse Anzahl von feinen Körnern der tigroidalen Substanz. Der Zellkern ist gross, deutlich und enthält ein zentral situierten Kernkörperchen.

23. Nucleus medianus posterior (pm - Abb. 6, 7 u. 32), auch Nucleus Edinger Westphal benannt, ist der Reihe nach der zweite Nucleus $n$. oculomotorii. Er ist ein kurzer, schmaler, ziemlich deutlich von der Umgebung abgesonderter Zellstreifen, der in der Nähe der Naht ventral von der Wasserleitung des Mittelhirnes, aber dorsal vom vorderen Abschnitt des Nuclei $n$. oculomotorii gelegen ist. In der Umgebung des vorderen Poles von Nucleus oculomotorii geht der erwähnte Kern stufenweise in den Nucleus medianus ant. über. Auf den Querstreifen ist der posteriore Abschnitt dieses Kernes eine kleine, abgerundete Zellgruppe. Im vorderen Abschnitt nimmt der Kern die Gestalt einer schmalen Zellgruppe an, die schräg von der dorso-lateralen Seite zur ventro-medialen gelegen ist. In diesem Abschnitt bilden Nucleus medianus post. mit dem gleichnami- 
gen Kern der gegenüber liegenden Seite eine Zellanhäufung, die ihrer Gestalt nach mit dem Bucbstaben „V" ähnelt. In der Umgebung des vorderen Poles von Nucleus n. oculomotorii geht der beschriebene Kern stufenweise in den Nucleus medianus ant. über.

Den Nucleus medianus posterior bilden gedrängt angeordnete, sich intensiv färbende, dreieckige, vielpolige und abgerundete Zellen mit Ausmassen von 10 bis $20 \mu$. Der Zellkern ist verhältnismässig gross, aber wenig deutlich mit einem zentral situierten Kernkörperchen. Die tigroidale Substanz ist in Gestalt von Körnern verschiedener Grösse auf der Peripherie der Zelle angehäuft.

24. Nucleus medianus anterior (am - Abb. 8 u. 33) ist eine Verlängerung in vorderer Richtung des vorherigen Kernes, von dem er sich durch den Zellbestand unterscheidet. Eine ziemlich deutliche Verengung teilt beide Kerne voneinander. Nucleus medianus ant. zieht sich auf der Strecke von der Umgebung des vorderen Poles von Nucleus n. oculomotorii bis zum vorderen Pol von Nucleus ruber entlang. Die Länge von Nucleus medianus ant. gleicht annähernd derjenigen von Nucleus mediauns post.

Auf den Querfortsätzen ist der erwähnte Kern eine kleine, schmale Zellgruppe, die parallel zur Naht in ihrer Nähe ventral und medial von Nucleus Darkschewitschi aber dorsal und medial von Nucleus ruber angeordnet ist. Der Kern ist deutlich, denn er wird von gedrängt anliegenden sich intensiv färbenden Zellen gebildet. Auf einigen Präparaten liegt der Nucleus medianus anterior sehr nahe an die mediale Fläche an und kontaktiert fast mit dem gleichnamiger. Kern der gegenüberliegenden Seite.

Den Kern bilden spindelförmige, abgerundete und unzahlreiche vielpolige Zellen mit Ausmassen von 20 bis $30 \mu$. Die spindelförmigen Zellen sind mit der Längsachse senkrecht angeordnet. Der Zellkern ist kugelig, nicht besonders deutlich mit einem zentral situierten Kernkörperchen. Die tigroidale Substanz ist in Gestalt von zahlreichen feinen Körnern auf der Peripherie der Zellen angehäuft. Die Zellen von Nucleus medianus ant. färben sich intensiver als diejenigen des Nucleus Darkschewitschi.

25. Nucleus ruber ( $\mathrm{nr}-\mathrm{Abb} .5--8$ u. 34) ist ein sehr deutlicher, langer Zellstreifen, der ventral und lateral von Fasciculus longitudinalis medialis, aber dorsal und medial von Pars compacta substantiae nigrae situiert ist. Der posteriore Pol von Nucleus ruber ist in ungrosser Entfernung nach vorn hin vom posterioren Pol des Nucleus $n$. oculomotorii gelegen. Der vordere Pol des erwähnten Kernes, der bis zum Zwischenhirn reicht, befindet sich auf der Höhe der Querfläche, die durch den postericren Pol von Corpus mamillare geführt ist.

Auf den Querschnitten hat Nucleus ruber die Gestalt einer grossen 
Zellgruppe, deren Form in den verschiedenen Abschnitten des Kernes veränderlich ist. Im posterioren Abschnitt erinnert er an ein gleichseitiges Dreieck mit abgerundeten Ecken, aber im anterioren Abschnitt ist die Gestalt des Kernes abgerundet. Auf einigen Präparaten weist $\mathrm{Nu}$ cleus ruber eine ziemlich deutliche Einteilung in zwei Teile auf den dorso-medialen und ventro-lateralen.

Den roten Kern bilden sich intensiv färbende dreieckige und vielpolige Zellen mit Ausmassen von 30 bis $70 \mu$. In der posterioren Hälfte des Kernes überwiegen grosse Zellen, jedoch in der anterioren besteht die Mehrzahl aus Zellen mit kleineren Ausmassen. Die Zellen im Kern sind unregulär angeordnet - dichter auf der Peripherie, aber loser in der Kernmitte. Sie sind mit zahlreichen, feinen Körnern der tigroidalen Substanz ausgefüllt. Der Zellkern ist gross, kugelig mit einem zentral situierten Kernkörperchen.

26. Nucleus pedunculi mamillaris (npm - Abb. 7, 8 u. 35) ist ein langer schmaler Zellstreifen, der im ventro-medialen Teil des vorderen $\mathrm{Ab}$ schnitten des Mittelhirnes gelegen ist. Der posteriore Pol des beschriebenen Kernes erscheint in kleiner Entfernung nach vorn hin von der Querfläche, die durch den posterioren Pol des roten Kernes geführt ist. Der vordere Pol von Nucleus pedunculi mamillaris befindet sich auf der Höhe des anterioren Poles von Nucleus basalis radicis optici.

Auf den Querschnitten ist die Gestalt des Kernes veränderlich. Der posteriore, kurze Abschnitt des Kernes ist eine kleine schmale Zellgruppe, die an der ventralen Fläche des Mittelhirnes gelegen ist und zwar medial von Pars compacta substantiae nigrae, aber lateral von Nucleus interpeduncularis. In diesem Abschnitt begrenzt der Kern den Pedunculus mamillaris von der ventralen Seite. In vorderer Richtung vergrössert sich der Kern und nimmt die Gestalt einer ziemlich breiten bogenförmig ausgebogenen Zellgruppe an, die den Pedunculus mamillaris von der ventro-medialen Seite umfasst. Gleichzeitig entfernt sich der Kern von der ventralen Fläche des Mittelhirnes ein klein wenig. Der vordere Abschnitt des Kernes ist weniger deutlich. Er wird von einer lose angeordneten Zellgruppe von unregulärer Gestalt gebildet, die sich zwischen der medialen Fläche und dem Nucleus basalis radicis optici, an der ventralen Fläche des Mittelhirnes befindet. In der Nähe des vorderen Poles verringert sich die Zellanzahl im Kern stufenweise und er unterliegt allmählich in der Umgebung der Querfläche, die durch den anterioren Pol von Nucleus basalis radicis optici durchgeführt wird, dem Schwund.

Den Nucleus pedunculi mamillaris bilden sich intensiv färbende dreieckige und vielpolige aber auch einige spindelförmige Zellen mit Ausmassen von 20 bis $40 \mu$. Die tigroidale Substanz hat eine schaumarti- 
ge Konsistenz. Der Zellkern ist gross, kugelig und enthält ein zentral - situjertes Kernkörperchen.

27. Nucleus Darkschewitschi ( $\mathrm{nD}$ - Abb. 7, 8 u. 36) ist ein schwer abzusondern der Zellstreifen, der im ventro-medialen Teil der grauen periventrikulären Substanz des vorderen Abschnittes des Mittelhirnes und des posterioren Abschnittes des Zwischenhirnes gelegen ist. Seine Gestalt und seine Grenzen sind schwer zu bestimmen. Der Darkschewitschi-Kern zieht sich ungefähr auf der Strecke vom vorderen Pol des Nucleus $n$. oculomotorii bis zum vorderen Pol von Nucleus medianus ant. lang. Er ist nur in seinem Mittelabschnitt deutlicher. Auf den Querschnitten hat dieser Kern in diesem Abschnitt die Gestalt einer ovalen schräg angeordneten Zellgruppe, die in dem ventro-medialen Teil der grauen periventrikulären Substanz, auf der dorso-medialen Seite von Nucleus interstitialis fasciculi longitudinalis med. und dorso-lateral von Nucleus medianus ant. gelegen ist.

Den Darkschewitschi-Kern bilden ziemlich lose angeordnete, sich nicht besonders intensiv färbende dreieckige, vielpolige, abgerundete und spindelförmige Zellen mit Ausmassen von 15 bis $30 \mu$. Die spindelförmigen Zellen sind weniger zahlreich. Verhältnismässig die grössten Ausmassen haben die dreieckigen und vielpoligen Zellen. Die tigroidale Substanz tritt in Form von wenigen ziemlich feinen Körnern auf. Der Zellkern ist kugelig, schwach sichtbar und enthält ein intensiv gefärbtes zentral situiertes Kernkörperchen.

28. Nucleus basalis radicis optici (bo - Abb. 8 u. 37) ist ein kurzer, schmaler aber deutlicher Zellstreifen, der im ventralen Teil des vorderen Abschnittes des Mittelhirnes, über dem medialen Rand des Pedunculus cerebri gelegen ist. Der posteriore Pol des beschriebenen Kernes befindet sich auf der Höhe der Querfläche, die durch den vorderen Pol von Nucleus n. oculomotorii durchgeführt wird. Vorn schwindet Nucleus ba.salis radicis optici auf der Höhe des anterioren Endes von Pars lat. substantiae nigrae.

Auf den Querschnitten hat der Kern die Gestalt einer schmalen Zellgruppe, die schräg von der dorso-lateralen Seite zur ventro-medialen Seite in der Nähe des medialen Randes von Pars compacta substantiae nigrae, lateral von Pedunculus mamillaris, aber ventral und lateral von Nucleus ruber gelegen ist. In der Nähe des anterioren und posterioren Poles verringert sich die Anzahl der Zellen im Nucleus basalis radicis optici schnell und der Kern unterliegt einem raschen Schwund.

Den Nucleus basalis radicis optici bilden gedrängt angeordnete, sich intensiv färbende dreieckige und vielpolige aber auch vereinzelte spindelförmige Zellen mit Ausmassen von 20 bis $50 \mu$. Die Zellen sind mit zahlreichen, groben Körnern der tigroidalen Substanz ausgefüllt. Der Zell- 
kern ist kugelig, undeutlich und enthält ein zentral situiertes Kernkörperchen. Die Zellen des erwähnten Kernes färben sich intensiver und sirıd dichter angeordnet als die Zellen der Pars compacta substantiae nigrae.

29. Nucleus interstitialis fasciculi longitudinalis medialis (nit - Abb. 8 u. 38), auch Nucleus interstitialis Cajali benannt, ist ein kurzer, nicht besonders deutlicher Zellstreifon, der dorsal und lateral von Fasc. longiiudinalis med., an der ventro-lateralen Fläche von Nucleus Darkschewitschi gelegen ist. Der erwähnte Kern zieht sich auf der Strecke von der Querfläche, die in der halben Entfernung zwischen dem vorderen Pol des Nucleus $n$. oculomotorii und dem vorderen Pol des roten Kernes geführt ist, bis zur Querfläche, die sich minimal in vorderer Richtung vom vorderen Pol des Nucleus medianus ant. befindet.

Auf den Querschnitten ist der beschriebene Kern als unregulär gestaltete Gruppe von sehr lose angeordneten Zellen sichtbar. In dem Mittelabschnitt des Kernes gibt es mehr Zellen und diese ordnen sich auf einem grösseren Raum an als im anterioren und posterioren Abschnitt.

Den Nucleus interstitialis fasciculi longitudinalis med. bilden sich intensiv färbende vielpolige, dreieckige aber auch vereinzelte spindelförmige Zellen mit Ausmassen von 20 bis $40 \mu$. Der Zellkern ist gross, kugelig, gut sichtbar und er enthält ein zentral situiertes Kernkörperchen. In den Zellen treten ziemlich zahlreiche, grobe Körner der tigroidalen Substanz auf. Die Zellen des beschriebenen Kernes färben sich dunkler als diejenigen des Nucleus Darkschewitschi aber ungefähr genau so wie diejenigen von Nucleus medianus ant.

30. Nucleus $X$ ( $\mathrm{nx}-\mathrm{Abb} .8$ u. 39) ist ein ziem! ich deutlicher Zellstreifen, der im Vorderabschnitt des Mittelhirnes und im posterioren Abschnitt des Zwischenhirnes, ventral und lateral von Fasc. longitudinalis med., aber dorsal und medial von Nucleus ruber gelegen ist. Posterior trscheint der Kern in der halben Länge von Nucleus ruber. In anteriorer Richtung reicht der erwähnte Kern weiter als Nucleus ruber und sein vorderer Pol reicht bis zur Querfläche, die mit dem anterioren Pol von Nucleus medianus ant. kontaktiert.

Auf den Querschnitten hat der Kern X im posterioren Abschnitt anfänglich die Gestalt einer schmalen aber später abgerundeten Zellgruppe, die zwischen dem Fasc. logitudinalis med. und dem Nucleus ruber gelegen ist. Der Mittelabschnitt des Kernes liegt in einer grösseren Entfernung vom roten Kern. In diesem Abschnitt hat Nucleus $X$ die Gestalt eines verlängerten Ovales, das schräg von der dorso-medialen Seite zur ventro-lateralen gelegen ist. In der Nähe des vorderen Poles verringert sich der Kern ziemlich schnell, nimmt dann auf den Querschnitten die Gestalt einer ventral und lateral von Fasc. longitudinalis med. gelegenen 
Zellgruppe an, unterliegt auf der Höhe des vorderen Poles von Nucleus medianus ant. dem Schwund.

Den Nucleus $X$ bilden, sich ziemlich schwach färbende, dreieckige, vielpolige und abgerundete Zellen mit Ausmassen von 15 bis $30 \%$. Der Zellkern ist rund, gut sichtbar und enthält ein zentral situiertes Kernkörperchen. Die Zellen enthalten unzahlreiche, feine Körner der tigroidalen Substanz. Die Zellen des Kernes X färben sich deutlich weniger intensiv als diejenigen von Nucleus medianus ant. und Nucleus ruber.

\section{BESPRECHUNG}

Eine weitgehende Diskussion auf das Thema des Schrifttums in Betrefí des Baues und der Topographie der Kerne des Mittelhirnes vom Sumpfbiber scheint überflüssig zu sein, denn dies wurde schon früher in Arbeiten anderer Autoren vollzogen. An dieser Stelle muss vor allen die Arbeit von Crosby \& Woodburne (1943) erwähnt werden, in der das Schrifttum bis zum Jahre 1943 genau durchdiskutiert wurde, weiter die Arbeit von Chomiak (1966), die die Beendigung eines Untersuchungszyklus dieses Autors über Bau und Topographie der Kerne im Mittelhirn der domestizierten Huftiere darstellt und eine genaue Besprechung des Schrifttums in Bezug des Mittelhirnes in der Zeitspanne der Jahre von 1943 bis 1965 enthält. Aus diesem Grunde begrenzte ich mich nur auf die Angabe der charakteristischesten Unterschiede, die in Lage, Gestaltung und Bau der Zellen einiger Kerne des Mittelhirnes des Sumpfbibers im Vergleich mit denselben Nervenzentren bei anderen Säugern beobachtet worden waren.

Die Vergleichung des Kernzusammenspiels der lateralen Schleife des Sumpfbibers mit demjenigen bei anderen Säugern ist in Hinsicht auf die besonders zahlreiche und verschiedenartige Nomenklatur, die durch verschiedene Autoren bei Beschreibungen angewandt worden ist, sehr erschwert. In vorliegender Arbeit ist die Nomenklatur nach Gillil a n \& Gels to n (1943) engewandt worden. Es scheint, dass die Kerne der lateralen Schleife beim Sumpfbiber eine grössere Ähnlichkeit mit den Kernen der lateralen Schleife der domestizierten Huftiere aufweisen (Gillilan \& Gelston, 1943; Chomiak, 1961; 1962; 1964; Cho$\mathrm{miak} \& \mathrm{~W}$ ele $\mathrm{nto}, 1963 ; 1964)$ als bei den Carnivoren ( $\mathrm{T} \mathrm{a} \mathrm{be} \mathrm{r,} \mathrm{1961;}$ Adrianov \& Mering, 1959). Die Nuclei lemnisci laterales des Sumpfbibers unterscheiden sich von denjenigen bei domestizierten Huftieren durch das Fehlen einer Einteilung bei Pars dorsalis nuclei caudalis ventralis lemnisci lateralis auf Pars dorso-caudalis und dorso-rostralis. Taber (1961) sonderte dagegen bei der Katze und Adrianov \& M e r ing (1959) bei dem Hund nur zwei Kerne der lateralen Schleife ab nämlich: die dorsalen und lateralen. Der erste von diesen beiden Ker- 
nen ist mit Nucleus dorsalis lemnisci lat. des Sumpfbibers gleichartig, aber der zweite entspricht Nucleus caudalis ventralis bzw. nur Pars ventro-medialis dieses Kernes beim Sumpfbiber. Es ist auch möglich, dass Nucleus paralemniscalis, der bei der Katze durch Taber (1961) beschrieben worden ist, mit Nucleus ventralis rostralis beim Sumpfbiber gleichartig ist. Eine ähnliche Gestaltung des Kernes der Seitenschleife wie bei der Katze und dem Hund stellten ebenfalls Me essen \& O ls ze w ski (1949) bei dem Kaninchen und auch W ünscher, Sch obe r \& W e rne r (1965) bei der Ratte fest.

Nucleus mesencephalicus radicis $n$. trigemini weist beim Sumpfbiber in Betreff seiner Lage und Gestaltung keine grösseren Unterschiede im Vergleich mit demselben bei anderen Säugern auf. Es muss aber vermerkt werden, dass dieser Kern beim Sumpfbiber nur durch eine Art von Zellen gebildet wird, aber Gillilan \& Gelst on (1943) stellten beim Schwein und Schaf und B r ow n (1943) beim Hund im Nucleus mesencephalicus radicis $n$. trigemini zwei Zelltypen fest. Bei der Katze wurden sogar in diesem Kern drei Zelltypen beschrieben (B row n, 1943).

Der Nucleus interpeduncularis ist beim Sumpfbiber im Vergleich mit der Mehrzahl der in dieser Hinsicht untersuchten Säuger ziemlich charakteristisch. T a be r (1961) bei der Katze, Ch om i a k $(1961,1962)$ beim Schaf und Schwein, Chomiak \& Welento (1964) beim Pferd stellten die Einteilung des Nucleus interpeduncularis in den medialen Teil, der von kleinen Zellen gebildet wird, und den lateralen fest, der seinerseits von bedeutend grösseren Zellen geformt ist. Bei der Katze kennzeichnet sich diese Einteilung nur im medialen Abschnitt des Kernes. Beim Sumpfbiber ist der Nucleus interpeduncularis einheitlich und weist keine Einteilung in einen lateralen und medialen Teil auf. Ein ähnliches Fehlen an Differenzierung des Nucleus interpeduncularis in einen lateralen und medialen Teil wie beim Sumpfbiber beobachteten $\mathrm{Ch}$ o m i a k \& W e le n to (1963) beim Rind.

Die Lage des Nucleus $n$. trochlearis weist eine ziemlich grosse Ungleichartigkeit bei den einzelnen Säugerarten auf. Bei dem Sumpfbiber ordnet sich dieser Kern auf der dorso-lateralen Seite von Fasc. longitudinalis med. an. Einen ähnlich situierten Nucleus $n$. trochlearis beschrieb T a ber (1961) bei der Katze und A d r i a n ov \& M e r ing (1959) beim Hund. Beim Pferd dagegen ( $\mathrm{Ch}$ om iak \& Welento, 1964; Gillil a n \& Gelston, 1943) und bei der Ratte (W ünscher et al., 1965) liegt er ventral, aber beim Schaf ( $\mathrm{Chomiak}$ 1961), beim Schwein (C hom i a k, 1962), bei der Ziege ( $\mathrm{Chomiak}, 1964)$ und beim Rind (Chomiak \& Welento, 1963) dorsal vom Fasc. longitudinalis med. Der Nucleus nervi trochlearis des Sumpfbibers ist ähnlich wie derselbe 
bei anderen Säugern aus grossen vielpoligen Zellen gebildet. Es ist jedoch erwähnenswert, dass z. B. dieser Kern bei der Ziege ( $\mathrm{C}$ h o m i a k, 1964) von abgerundeten und birnenförmigen Zellen gebildet wird und vielpolige Zellen sind verhältnismässig in geringer Anzahl vorhanden. Der vordere Pol des Nucleus $n$. trochlearis des Sumpfbibers vereinigt sich rnit dem posterioren Pol von Nucleus n. oculomotorii, während bei der Ratte (W ü $\mathrm{sch}$ e r et al., 1965) zwischen beiden Kernen eine deutliche Unterbrechung besteht.

Als charakteristisches Merkmal zeigt sich beim Nucleus linearis des Sumpfbibers seine verhältsmässig schwache Ausgestaltung im Vergleich mit demselben Kern bei anderen Säugern. Es muss ebenfalls unterstrichen werden, dass man im Nucleus linearis des Sumpfbibers nur zwei Teile absondern kann: den anterioren und posterioren, während z. B. Chomiak (1961, 1962, 1964), Chomiak \& Welento $(1963,1964)$ und Gillilan \& Gelston (1943) bei domestizierten Huftieren, aber auch B rown (1943) bei der Katze und dem Hund eine Einteilung dieses Kernes in drei Teile festgestellt haben: den anterioren, medialen und posterioren. Es muss aber zugegeben werden, dass $\mathrm{T}$ a be $\mathrm{r}$ et al. (1960), die ebenfalls den Nucleus linearis bei der Katze untersucht haben, in ihm zwei Anhäufungen abgesondert haben nämlich: Nucleus linearis intermedius und Nucleus linearis rostralis. Der erste von diesen Kernen scheint Pars caudalis, der zweite aber Pars oralis nuclei linearis des Sumpfbibers zu entsprechen.

Bei dem Sumpfbiber begrenzt Nucleus annularis fasciculi longitudinalis med. den Fasc. longitudinalis med. nur von der ventro-medialen Seite so wie dies auch ähnlich bei den domestizierten fiuftieren stattfindet (Chomiak, 1961, 1962, 1964; Chomiak \& Welento, 1963, 1964; Gillilan \& Gelston, 1943) und beim Hund (Brown, 1943). Anders gestaltet ist dieser Kern bei der Katze ( $\mathrm{T}$ a b e r, 1951) und beim Menschen ( $\mathrm{R}$ i l e y, 1960 u. a.), bei denen er den Fasc. longitudinalis med. rings herum umfasst.

Die schwarze Substanz des Sumpíbibers mit einem eben solchen Nervenzentrum bei anderen Säugern vergleichend, scheint es, dass diese ihrer Gestaltung nach am meisten an die Substantia nigra bei der Katze ( $\mathrm{T}$ a b e r, 1961) angenähert ist. Ähnlich wie bei diesem letzten Tier ist von den drei Teilen (kompakter, retikulärer und lateraler) der schwarzen Substanz bei dem Sumpfbiber der kompakte Teil am längsten, aber am kürzesten und am schwächsten ausgestaltet ist die Pars lat. Eine derartige Gestaltung der schwarzen Substanz beim Sumpfbiber weist im Vergleich mit eirigen anderen Säugern ziemlich grosse Unterschiede auf. So haben z. B. K r i e g (1948) bei Macacus rhesus und W ü n s h e r et al. (1965) bei der Ratte festgestellt, dass die schwarze Substanz nur aus zwei 
Teilen besteht nämlich: dem kompakten und retikulären, aber bei dem Elefanten (V e r h a a r t, 1962) ist die Pars compacta beträchtlich kürzer und schlechter ausgestaltet als Pars reticularis.

Den roten Kern, der von vielen Autoren als ein Teil der retikulären Substanz betrachtet wurde (B odal, 1958 u. a.) begann man in einen klein- und grosszelligen Teil einzuteilen. Diese Eirteilung wurde zum ersten Mal durch Hat s che k (1907) bei seinen Vergleichsuntersuchungen über den roten Kern durchgeführt. Spätere Untersuchungen anderer Autoren erwiesen jedoch, dass solch eine Einteilung nicht bei allen Säugern schematisch angewandt werden kann. Schon Gillilan \& Gels to n (1943) haben festgestellt, dass die Einteilung von Nucleus ruber auf Pars parvo- und magnocellularis bei den domestizierten Huftieren schwach gekennzeichnet ist. Diese Beobachtungen wurden durch die Untersuchungen von $\mathrm{Chomiak}(1961 ; 1962)$ und $\mathrm{Chomiak} \&$ Welento $(1963 ; 1964)$ bestätigt, aus denen hervorging, dass man unter den Haustieren nur beim Pferd und Schaf eine schwach angedeutete Differenzierung des roten Kernes auf einen klein- und grosszelliger Teil beobachten konnte, aber dass beim Rind und Schwein solch eine Einteilung des Nucleus ruber überhaupt nicht auftritt. W ü n s c h e r et al. (1965), haben ebenfalls im roten Kern der Ratte keinen klein- und grosszelligen Teil abgesondert. Im Nucleus ruber des Sumpfbibers ist die Einteilung in Pars parvo- und magnocellulare ebenfalls nicht gekennzeichnet.

\section{SCHRIFTTUM}

1. Adrianov O. \& Mering T., 1959: Atlas mozga sobaki. Gos. izd. med. lit.: 52-69. Moskva.

2. Brodal A., 1958: The reticular formation of the brain stem. Anatomical aspects and functional correlations. Oliver and Boyd: 17-56. Edinburgh.

3. Brown J., 1943: The nuclear pattern of the non-tectal portions of the midbrain and isthmus in the dog and cat. J. comp. Neurol., 78: 365-405.

4. Ch o mi a k M., 1961: Jądra śródmózgowia owcy. Annls Univ. M. Curie-Skłodowska, DD 14 (1959): 147-187.

5. Ch omiak M., 1962: Jądra śródmózgowia świni. Ibidem, DD 15 (1960): 33-64.

6. Chom i a k M., 1964: Kerne des Mesencephalon der Ziege. Ibidem, DD 18 (1962): $19-36$.

7. Chom i a k M., 1966: Abschliessende Besprechung. Ibidem, DD 20 (1965): 69-92.

8. Chom iak M. \& W elento J., 1963: Kerne des Mesencephalon der Kuh. Ibidem, DD 16 (1961): 45-66.

9. Chomiak M. \& W elento J., 1964: Kerne des Mesencephalon beim Pferd. Ibidem, DD 17 (1962): 45-64.

10. Crosby E. \& Woodburne R., 1943: Discussion of the literature. J. comp. Neurol., 78: 253-288.

11. Gillilan L. \& Gelst on L., 1943: The nuclear pattern of the midbrain and isthmus in Ungulaten. J. comp. Neurol., 78: 289-364.

12. H a t s hek R., 1907: Zur vergleichenden Anatomie des Nucleus ruber. Neurol. Inst. an der Wiener Univ., 15: 89-136. 
13. Krieg W., 1948: A reconstruction of the diencephalic nuclei of Macacus rhesus. J. comp. Neurol., 88: 1-51.

14. M e essen H. \& Olszewski J., 1949: Cytoarchitektonischer Atlas des Rautenhirn des Kaninchens. S. Karger: 35-40. Basel- New York.

15. R i le y H., 1960: An atlas of the basal ganglia, brain stem and spinal cord. Hafner Publishing Co: 98-167. New York.

16. Szte yn S., 1963: Jądra ruchowe rdzenia przedłużonego nutrii. Annls Univ. M. Curie-Skłodowska, DD 16 (1961): 119-134.

17. S zt e y n S., 1964: Jądra czuciowe rdzenia przedłużonego nutrii. Ibidem, DD 17 (1962): $21-43$.

18. Szte y n S., 1965: Oliwa tylna (nucleus olivaris caudalis) nutrii. Ibidem, C 19 (1963): 177-188.

10. S z t e y n S., 1966: Budowa i topografia jąder móżdżku nutrii. Pol. Arch. wet., 10: $309-320$.

20. T a ber E., 1961: The cytoarchitecture of the brain stem of the cat. I. Brain stem nuclei of cat. J. comp. Neurol., i16: 27-70.

21. Taber E., Brodal A. \& Walberg F., 1960: The raphe nuclei of the brain stem in the cat. J. comp. Neurol., 114: 239-282.

22. Verhaart W. J. C., 1962: Anatomy of the brain stem of the Elephant. J. f. Hirnforsch., 5: 455-524.

23. Wünscher W., Schober W. \& Werner L., 1965: Architektonischer Atlas von Hirnstamm der Ratte. S. Hirzel Verlag, 30-59. Leipzig.

Eingang des Ms., 11. März 1967.

Department of Animal Anatomy,

College of Agriculture,

Lublin, Akademicka 11, Poland.

Stanisław SZTEYN

BUDOWA I TOPOGRAFIA JĄDER NERWOWYCH SRODMOZGOWIA NUTRII

\section{Streszczenie}

Badania zostaly wykonane na podstawie skrawków poprzecznych (grubości $15 \mu$ ) mózgowi 11-miesięcznych nutrii. Skrawki barwiono wg metody Nissla. W pracy opi-sano położenie, ukształtowanie i budowę komórkową jąder nerwowych śródmózgou:ia nutrii (rys. 1-39). Spośród jąder nerwowych śródmózgowia nutrii tylko niektóre wỵkazują różnice w porównaniu z takimi samymi ośrodkami nerwowymi innych ssaków. W zespole jąder wstęgi bocznej nutrii cechą charakterystyczną jest brak podziału pars dorsalis nuclei caudalis ventralis lemnisci lateralis na pars dorso-caudalis i pars dorso-rostralis. Jądro międzykonarowe jest u nutrii jednolite i różni się tym od większości ssaków, u których ten ośrodek nerwowy wykazuje wyraźny podział na część boczną i środkową. Jądro liniowe nutrii jest stosunkowo słabo wykształcone i można w nim wyróżnić jedynie dwie części: przednią i tylną, podczas gdy $u$ innych gatunków zwierząt ssących podzielone jest ono $z$ reguły na trzy części: przednią, środkową i tylną. Jądro nerwu bloczkowego usytowane jest po grzbietowo-bocznej stronie fasciculus longitudinalis medialis, a jego biegun przedni połączony jest $z$ tylnym biegunem jądra głównego nerwu okoruchowego. Spośród 
trzech części istoty czarnej u nutrii najdłuższa jest pars compacta, a najkrótsza i najsłabiej wyksztalcona pars lateralis. Jądro pierścienne pęczka podłużnego przyśrodkowego ogranicza fasciculus longitudinalis medialis jedynie od strony brzusznoprzyśrodkowej. Jądro czerwienne nie wykazuje zróżnicowania na pars parvo- i magnocellularis.

\section{TAFELBESCHREIBUNG}

Tafel $\mathbf{X}$.

Querschnitte des Gehirnes des Sumpfbibers in verschiedenen Umgebungen (Vergr. 5,5 $\times$ ).

Abb. 1. Umgebung des posterioren Poles von Nucleus latero-dorsalis tegmenti. Abb. 2. Umgebung des posterioren Poles von Nucleus medialis prof.

Abb. 3. In der Nähe des posterioren Poles von Nucleus ventralis rostralis lemnisci lat.

Abb. 4. In der Nähe des posterioren Poles von Nucleus n. trochlearis.

Abb. 5. In der Nähe des posterioren Poles von Nucleus ruber.

Abb. 6. In der Nähe des anterioren Poles von Nucleus interpeduncularis.

Abb. 7. In der Nähe des anterioren Poles von Nucleus $n$. oculomotorii.

Abb. 8. In der Nähe des anterioren Poles von Nucleus ruber.

Zellen der einzelnen Nervenkerne des Mittelhirnes des Sumpfbibers (Vergr. 225×).

\section{Tafel XI.}

Abb. 9. Pars ventro medialis nuclei caudalis ventralis lemnisci lateralis.

Abb. 10. Pars dorsalis nuclei caudalis ventralis lemnisci lateralis.

Abb. 11. Nucleus dorsalis lemnisci lateralis.

Abb. 12. Nucleus ventralis rostralis lemnisci lateralis.

Abb. 13. Nucleus dorsalis tegmenti.

Abb. 14. Nucleus mesencephalicus radicis nervi trigemini.

Abb. 15. Nucleus marginalis pedunculi cerebelli oralis.

Abb. 16. Nucleus latero-dorsalis tegmenti.

Abb. 17. Nucleus mesencephalicus profundus.

Abb. 18. Nucleus dorsalis raphe.

Abb. 19. Pars caudalis nuclei linearis.

Abb. 20. Pars oralis nuclei linearis.

Tafel XII.

Abb. 21. Nucleus annularis fasciculi longitudinalis medialis.

Abb. 22. Nucleus medialis profundus.

Abb. 23. Area cuneiformis.

Abb. 24. Nucleus interpeduncularis.

Abb. 25. Nucleus nervi trochlearis.

Abb. 26. Pars compacta substantiae nigrae.

Abb. 27. Pars reticularis substantiae nigrae.

Abb. 28. Pars lateralis substantiae nigrae.

Abb, 29. Nucleus ventralis tegmenti. 
Tafel XIII.

Abb. 30. Nucleus interstitialis commissurae Foreli.

Abb. 31. Nucleus nervi oculomotorii.

Abb. 32. Nucleus medianus posterior.

Abb. 33. Nucleus medianus anterior.

Abb. 34. Nucleus ruber.

Abb. 35. Nucleus pedunculi mamillaris.

Abb. 36. Nucleus Darkschewitschi.

Abb. 37. Nucleus basalis radicis optici.

Abb. 38. Nucleus interstitialis fasciculi longitudinalis medialis.

Abb. 39. Nucleus $X$. 


\section{ABKÜRZUNGERKLÄRUNG}

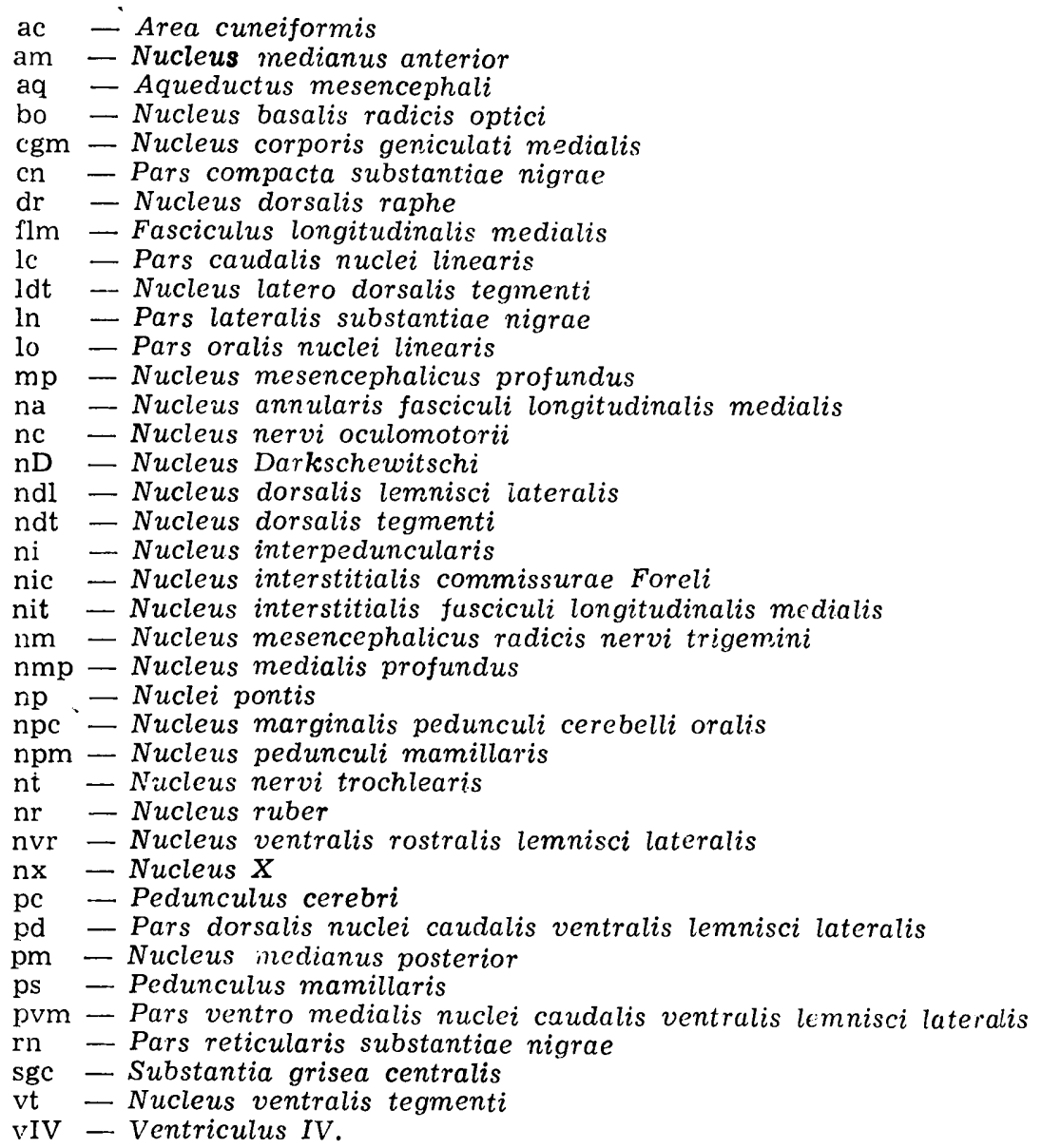




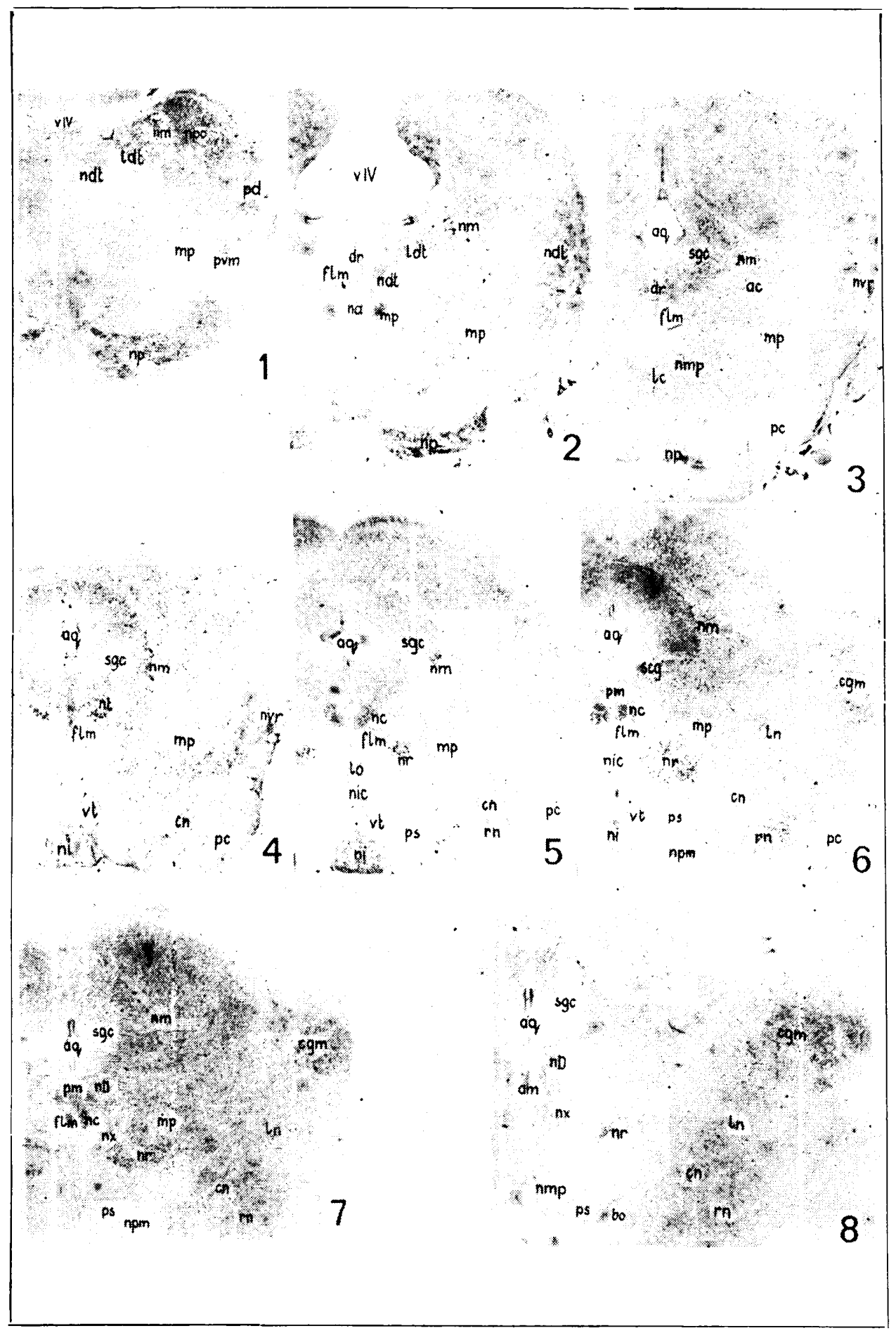




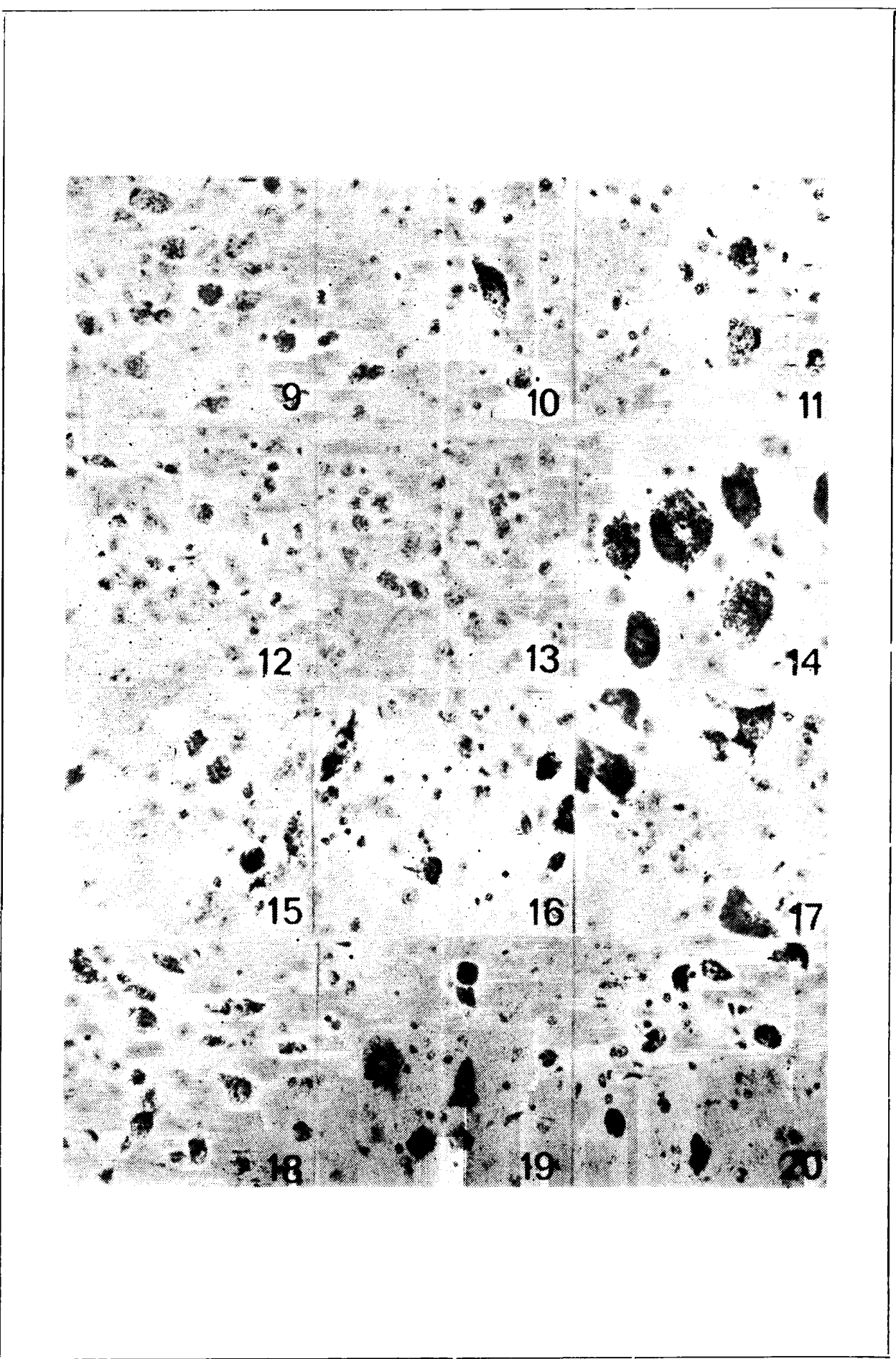




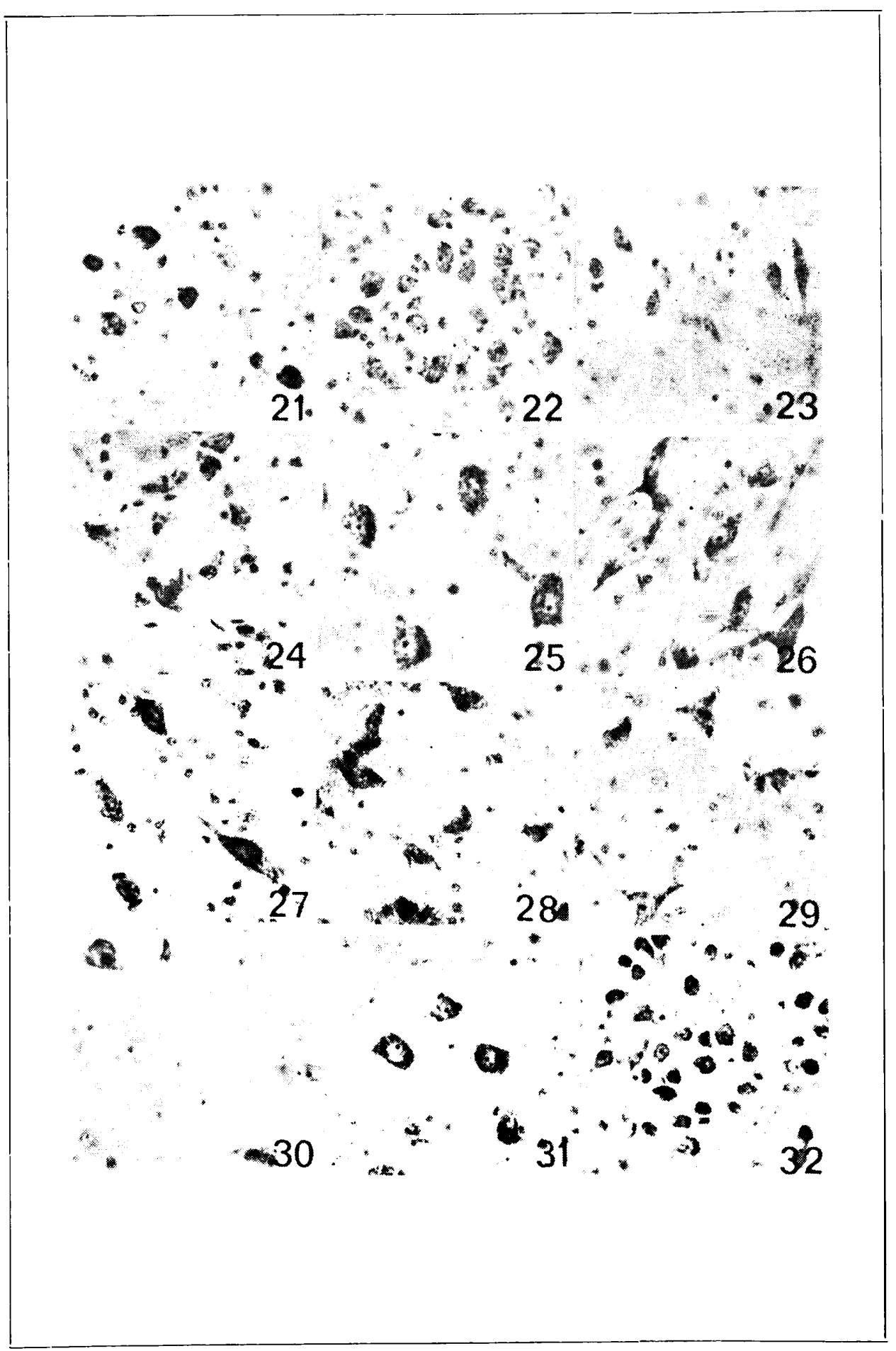




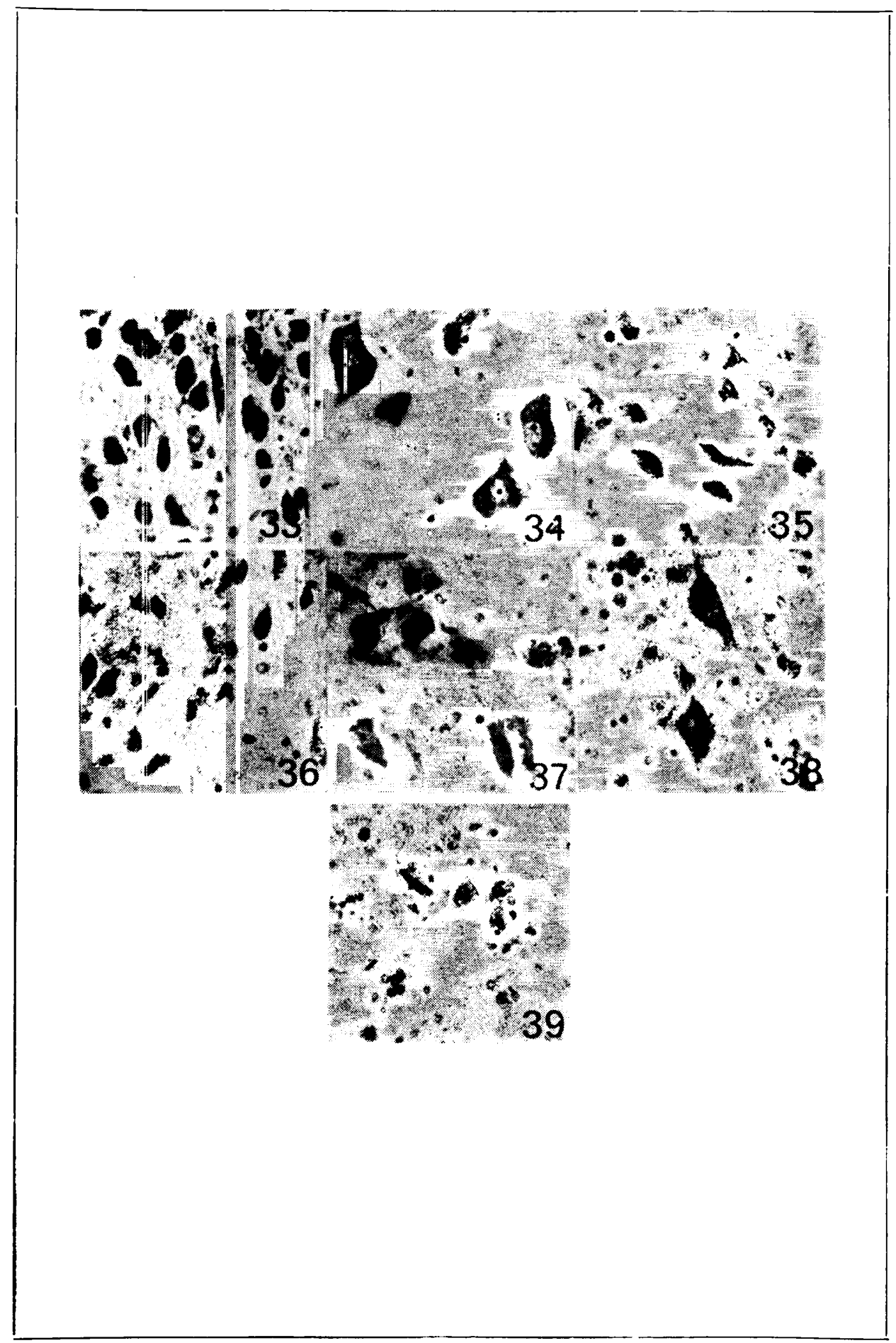

